\title{
Deficiency of laryngeal collagen type II in an infant with respiratory problems
}

\author{
K Frenzel, G Amann, B Lubec
}

\begin{abstract}
A dysmorphic infant is described who presented with laryngeal collapse leading to intubation and respiratory problems that were assigned clinically to the Sussman syndrome. The baby had repeated episodes of respiratory distress necessitating assisted ventilation. At 6 months old, uvulopharyngopalatotomy was done to enlarge the supraglottic airway without any benefit. Surgical reduction of the tongue and cricoid splitting did not ameliorate the respiratory distress; repeated extubation attempts failed with the baby developing stridor, respiratory distress, and episodes of cardiac arrest. At 10 months old he developed seizures and computed tomography showed diffuse cerebral atrophy consistent with hypoxicischaemic damage. He died at 17 months old. Western blots using antibodies against collagen $\alpha_{1}$ (II) showed an absence of collagen type II in laryngeal tissue, which may explain the laryngeal collapse and impaired respiratory functions. (Arch Dis Child 1998;78:557-559)
\end{abstract}

Keywords: larynx; collagen type II; dysmorphism; western blot

Among the many inherited deficiencies of collagen only a few disorders with absence of individual chains have been described. Missing collagen chains have been reported for skin and bone; generally the $\alpha_{1}$ or $\alpha_{2}$ chains of collagen type I were affected, although collagen type II and III defects have been reported.

Collagen type II defects have been found in chondrodysplasias ${ }^{1}$ with changes of electrophoretic mobility. Mutations of the type II procollagen gene (col2A1) have been found in Stickler syndrome or hereditary arthroophthalmopathy, ${ }^{2}$ and in the Sussman syndrome. ${ }^{3}$ Absence of collagen type II has only been reported in one case, a patient with Langer-Saldino achondrogenesis. ${ }^{4}$

We observed a lack of collagen type II in the

University of Vienna, Department of Neonatology,

Waehringer Guertel 18, A 1090 Vienna, Austria

K Frenzel

G Amann,

B Lubec

Correspondence to: Dr Lubec.

Accepted 3 November 1997 two siblings were apparently healthy. Birth weight was $2490 \mathrm{~g}$, length at birth was $45 \mathrm{~cm}$, and head circumference was $34 \mathrm{~cm}$. The infant
A boy was born spontaneously at 34 weeks' gestation to healthy, non-consanguineous, white parents following an uneventful pregnancy. Family history was unremarkable and developed respiratory distress syndrome, confirmed radiologically, requiring assisted ventilation for 48 hours. Subsequent feeding problems and pneumonia delayed discharge for one month.

The baby was readmitted four weeks later with failure to thrive, fever, a systolic murmur, and recurrent respiratory distress culminating in respiratory failure and the need for assisted ventilation. Echocardiography showed a dysplastic mitral valve and moderate hypertrophic cardiomyopathy; chest radiography showed pneumonia and increasing respiratory distress. Repeated attempts to extubate the child resulted in stridor and increasing respiratory failure. Laryngoscopic examination was normal. At 6 months old, uvulopharyngopalatotomy was done to enlarge the supraglottic airway without any benefit.

In addition to persistent respiratory problems the child had dysmorphic features including short stature, short neck, coarse facial features, enlargement of the tongue, ptosis of the left eye, short limbs with thick, tapering fingers, redundant skin over the palms and feet, puffiness over the dorsum of the hand, hypoplastic toenails, and floppiness.

Surgical reduction of the tongue and cricoid splitting did not ameliorate the respiratory distress; repeated extubation attempts failed with the baby developing stridor, respiratory distress, and episodes of cardiac arrest. At 10 months old he developed seizures and computed tomography showed diffuse cerebral atrophy consistent with hypoxic-ischaemic damage. He died at 17 months old.

Based on clinical symptoms and phenotype we diagnosed Sussman syndrome, ${ }^{3}$ although lack of segmentation of the thoracolumbar vertebrae, metaphyseal modelling abnormalities, and small secondary ossification centres located abnormally to the metaphyses were not found on radiography up to 6 months of age.

\section{Investigations}

The larynx, freed from adjacent connective tissue, was obtained at necropsy. It was kept frozen at $-70^{\circ} \mathrm{C}$ until sample preparation for biochemical studies, minced to small pieces, washed in isotonic saline, homogenised in an ice bath, and acid salt soluble collagen was extracted. Homogenised material was incubated in $0.05 \mathrm{M}$ acetic acid containing $0.005 \mathrm{M}$ EDTA and $5 \mathrm{mg}$ pepsin (Merck, Darmstadt, Germany) per $100 \mathrm{mg}$ (wet weight) tissue.

The tissue was incubated for 72 hours at $4^{\circ} \mathrm{C}$ on a shaker. The samples were spun down in a refrigerated centrifuge at $4000 \times g$, the 
A

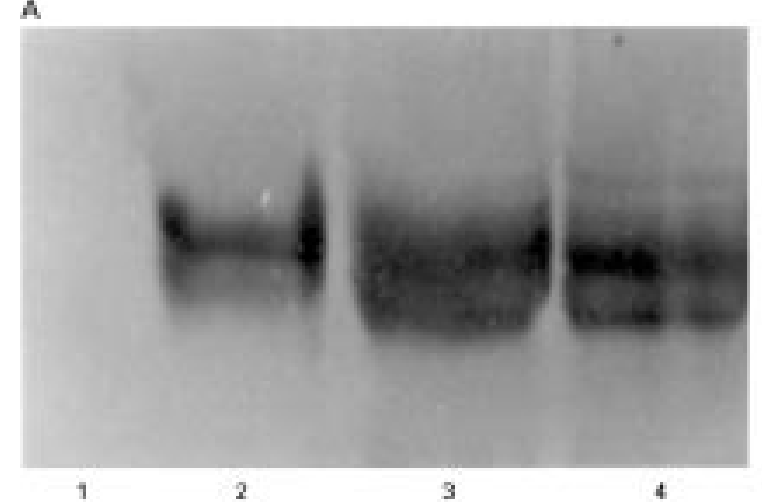

B

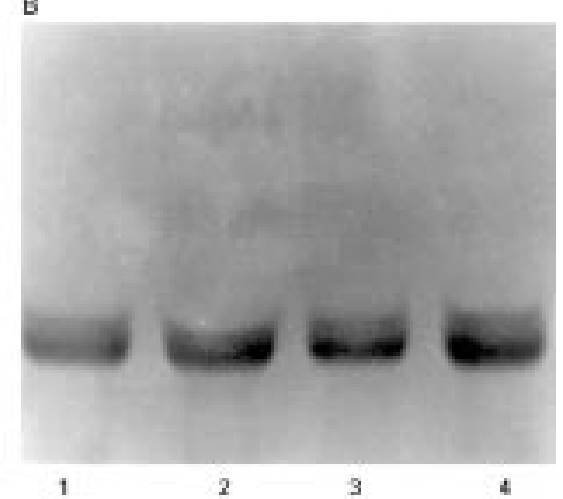

Figure 1 (A) The collagen extract from the patient (lane 1) lacks the $a_{1}$ (II) band on western blots, which is present in the three control extracts (lanes 2-4). (B) Western blot showing the collagen $a_{1}$ (I) band in the patient (lane 1) and three age matched controls (lanes 2-4).

supernatant was filtered through Millipore filters, and the protein concentration measured by the method of Bradford using a commercially available colorimetric assay (BioRad, Laboratories, Munich, Germany).

SDS polyacrylamide gel electrophoresis was done as described previously. ${ }^{5}$ Protein $(100 \mu \mathrm{g})$ was put in each well and gels were stained with a commercially available silver stain kit (BioRad Laboratories). Corresponding triplicates were unstained and transferred to nylon membranes for western blotting. Western blots were done according to a standard method. ${ }^{5}$ Polyclonal antibodies against collagens type I $\left(\alpha_{1}\right.$ (I)) and II (Institut Pasteur, Paris, France) were used.

MEASUREMENT OF LARYNX COLLAGEN

After mechanical removal of adjacent tissue, laryngeal tissue was defatted by diethylether: chloroform (1:1) and freeze dried. The dry tissue was weighed and 4-hydroxyproline was measured by the method of Woessner. ${ }^{6}$ The hydroxyproline content per milligram of larynx tissue was calculated and considered to reflect total collagen content. Laryngeal tissue from three age matched children was used for comparison of collagens type I and II, and for quantitative collagen studies.

\section{Results}

Western blots of extracted collagens from our patient showed an absence of the $\alpha_{1}$ (II) band (fig 1A). The band assigned to collagen $\alpha_{1}$ (I) was found in laryngeal tissue from our patient comparable to the three control samples (fig 1B).

\section{QUANTITATIVE STUDIES}

The mean (SD) 4-hydroxyproline content of the patient's larynx was less than for the three control children: 17 (1.3) $\mu \mathrm{g} / \mathrm{mg}$ laryngeal tissue for the patient; 49 (7.9) $\mu \mathrm{g} / \mathrm{mg}$ for the three controls.

\section{Discussion}

This is the first report of an absent collagen type II chain in the larynx. Mechanoelastic properties changed by the absence of this structural protein may well be compatible with impaired laryngeal function. Mechanisms lead- ing to absent collagen chains in general have been described and range from mutations to impaired post-transcriptional modifications and impaired secretion. Our patient had normal morphology by radiography; therefore, we suggest that the larynx was the only tissue with collagen type II deficiency, at least at the time of examination.

Kratochwil et al showed that retrovirus induced insertional mutation in mov13 mice affects collagen expression in a tissue and chain specific manner, ${ }^{7}$ and this model could explain our findings of tissue (and chain) specificity.

Another tentative mechanism for the absence of collagen type II exclusively from the larynx could be explained by the observations of Ryan and Sandell who showed that there were different mRNA populations for collagen type II. ${ }^{8}$ Metsaranta et al generated transgenic mice by microinjection of a $39 \mathrm{~kb}$ mouse pro $\alpha_{1}$ (II) collagen gene construct with a deletion of exon 7 and intron 7. ${ }^{9}$ This mutation was expected to disturb the assembly and processing of the homotrimeric type II collagen molecule in cartilage. The result of this genetic manipulation was a severe chondrodysplastic phenotype with short limbs, hypoplastic thorax, and abnormal craniofacial development; the affected pups died at birth from respiratory distress. We postulate that the deletion in the $\alpha_{1}$ (II) collagen acts as a dominant negative mutation disrupting the assembly and secretion of type II collagen molecules. The transgenic mice had exactly the same phenotype and clinical appearance as our patient, but the authors did not investigate the larynx of the transgenic model.

The possibility that collagen type II was not expressed in the larynx during early infancy can be ruled out, as Cohen et al demonstrated that type II collagen appeared soon after birth with levels increasing rapidly during the first months. ${ }^{10}$ The finding of a specific structural (biochemical) defect of laryngeal tissue in a dysmorphic child stimulated us to propose this mechanoelastic-biochemical link.

1 Murray LW, Bautista J, James PL, Rimoin DL. Type II collagen defects in the chondroplasias. Am $\mathcal{F}$ Human Genet 1989:45.5-15.

2 Knowlton RG, Weaver EJ, Struyk AF, et al. Genetic linkage of hereditary arthro-ophthalmopathy (Stickler syndrome) 
and the type II procollagen gene. Am $\mathcal{f}$ Hum Genet 1989;45:681-8.

3 Sussman MD, Kelly T, Rosenbaum KN, Balian G. Abnormality of cartilage collagen in a patient with unclassified chondrodystrophy. F Orthop Res 1984;2:339-45.

4 Feshchenko SP, Rebrin IA, Sokolnik VP, Sher BM, Kalinin VN, Lazjuk GI. The absence of type II collagen and changes in proteoglycan structure of hyaline cartilage in a case of Langer-Saldino achondrogenesis. Hum Genet 1989; 82:49-54.

5 Lubec B, Weninger M, Popow C, Vierhapper H, Lunec J, in patients with diabetes mellitus. Amino Acids 1993;4:11119.

6 Woessner JF. Determination of hydroxyproline in tissue and protein sample containing small proportions of this amino acid. Arch Biochem Biophys 1961;93:440-7.
7 Kratochwil, K, von der Mark, K, Kollar, et al. Retrovirus induced insertional mutation in mov 13 mice affects collagen I expression in a tissue specific manner. Cell 1989;57:807-16.

8 Ryan MC, Sandell LJ. Differential expression of a cysteine rich domain in the amino terminal propeptide of type II (cartilage) procollagen by alternative splicing of mRNA. 7 Biol Chem 1990;265:10334-9.

9 Metsaranta M, Garofalo S, Decker G, Rintala M, de Crombrugghe $\mathrm{B}$, Vuorio $\mathrm{E}$. Chondrodysplasia in transgenic mice harboring a 15-amino acid deletion in the triple helical domain of pro alpha 1 (II) collagen chain. f Cell Biol 1992; 118:203-12.

10 Cohen SR, Cheung DT, Lian G, et al. Collagen in the developing larynx. Ann Otol Rhinol Laryngol 1992;101: 328-33.

\section{Coagulation tests in inflicted head injury}

When child abuse is suspected the finding of abnormal coagulation test results may be seized upon by the defence as evidence of pre-existing bleeding disorder. Now data from Denver, Colorado (Kent P Hymel and colleagues, Pediatrics 1997;99:371-5) suggest that inflicted head trauma may cause such abnormalities.

The records of 265 children with inflicted head injury were reviewed and adequate data were available from 147 of whom 101 had computed tomography and/or magnetic resonance imaging evidence of injury to brain substance (IBS). A mildly prolonged prothrombin time was found in $54 \%$ of patients with and $20 \%$ without IBS. Median prothrombin time (normal 11.8 $+/-1.0)$ was 13.1 with IBS and 12.0 without. Presumptive evidence of disseminated intravascular coagulation was found in $37 \%$ of IBS and $7 \%$ of non-IBS patients. Mortality in IBS children was $32 \%$ and of those who died $94 \%$ had prolonged prothrombin time and $63 \%$ evidence of disseminated intravascular coagulopathy.

Head injury in children may cause coagulation test abnormalities and the medicolegal significance of this finding needs to be acknowledged. 